\title{
UNA APROXIMACIÓN A LA EXPERIENCIA DE VIVIR CON UN MENOR CON TRASTORNO DEL ESPECTRO AUTISTA DESDE LA NARRATIVA FAMILIAR DE LOS ADULTOS SIGNIFICATIVOS
}

\author{
AN APPROXIMATION TO THE EXPERIENCE OF LIVING WITH A \\ CHILD DIAGNOSED WITH AUTISM SPECTRUM DISORDER \\ THROUGH THE FAMILY NARRATIVE OF SIGNIFICANT ADULTS
}

Recibido: 16 de octubre de 2018 | Aceptado: 7 de noviembre de 2019

\begin{abstract}
EDUARDO M. M. TRONCOSO 1, ANA J. FERNÁNDEZ 2, CATALINA I. SAAVEDRA 3, CATALINA DE L. ORÓSTICA 4, CÉSAR A. VALENZUELA 5, CHRISTIAN F. VILLEGAS 6
\end{abstract}

1., 2., 3., 4., 5., 6., UNIVERSIDAD DE LA FRONTERA, Temuco, Chile

\begin{abstract}
Purpose: The purpose of this research was to describe the experience of significant adults of a family that lives with a member diagnosed with a developmental disorder, part of the Autism Spectrum Disorder (ASD), from the narrative of the other members. Method: As data recollection technique, a focal group interview was implemented to the family group which are the study sample, under a unique case design. The results were obtained through thematic analysis, using three main codes: knowledge and information, affectivity, and relationship dynamics. Results: As relevant findings of the research we can point out that changes produced in the family group by the diagnosis, were not consequent with the theory, as knowledge the diagnosis does not lead to a crisis, but it rather produced relief linked to the possibility of understanding the reality. Likewise, the family recognizes as relevant the professional support and information that experts could provide. Conclusion: it is positive for the family with a member with ASD to maintain permanent advice with a specialized team, avoiding isolation and strengthening the capacity to receive, understand and develop support resources.
\end{abstract}

KEY WORDS: Family narrative, Autism Spectrum Disorder, thematic analysis, family.

\section{CÓMO CITAR / HOW TO CITE}

Troncoso, E. M. M., Fernández A. J., Saavedra, C. I., Oróstica C. D. L., Valenzuela, C. A., Villegas, C. F. (2019). Una aproximación a la experiencia de vivir con un menor con trastorno del espectro autista desde la narrativa familiar de los adultos significativos. Salud \& Sociedad, 10(3), 210-225. doi: 10.22199/issn.0718-7475-2019-03-013

\footnotetext{
Correspondencia sobre este trabajo de be ser enviada a Eduardo Troncoso, Departamento de Psicología, Universidad de La Frontera, Francisco Salazar 01145, Temuco, Chile. Teléfono de contacto: 452325605 Correo de contacto: eduardo.troncoso@ufrontera.cl

1. Psicólogo, Magíster en Ciencias Sociales Aplicadas, Supervisor Clínico Acreditado y Especialista en Psicoterapia, docente de pre y post grado, Univer sidad de La Frontera. E-mail: eduardo.troncoso@ufrontera.cl; 2. Licenciada en Psicología, Universidad de La Frontera. E-mail: a.fernandez07@ufromail.cl; 3. Licenciada en Psicología, Universidad de La Frontera. E-mail: c.orostica01@ufromail.cl; 4. Licenciada en Psicología, Universidad de La Frontera. E-mail: c.saavedra06@ufromail.cl; 5. Licenciado en Psicología, Universidad de La Frontera. E-mail: c.valenzuela07@ufromail.cl; 6. Licenciado en Psicología, Universidad de La Frontera. E-mail: c.villega s02@ufromail.cl
} 
La Familia, Significados y Funciones

La familia es un grupo social unido por vínculos de consanguinidad, filiación y alianza (Bernales, 1993), una organización social primaria caracterizada por los lazos y relaciones afectivas entre sus miembros. Esta organización constituye un sistema, conformado por seres complejos con una finalidad en común: el sistema familiar es más que la suma de cada uno de sus integrantes como individuos.

El desarrollo de las familias varía según parámetros culturales, pero posee características universales. De acuerdo a Minuchin (1997), la familia es un sistema abierto en transformación, es decir, que constantemente recibe y envía descargas de y hacia el medio extrafamiliar y se adapta a las distintas demandas de la etapa en la que se encuentra. Asimismo, la familia muestra un desarrollo, que contempla etapas que exigen una reestructuración. Finalmente, la familia se adapta a las circunstancias cambiantes, manteniendo una continuidad y fomentando el crecimiento psicosocial de cada miembro. Por tanto, es una unidad social de estructura compleja debido a las relaciones que se producen en su interior.

Las funciones de la familia sirven a dos objetivos distintos. Uno interno, que refiere a la protección psicosocial de sus miembros, y otro externo, que refiere a la acomodación a una cultura y la transmisión de ésta (Minuchin, 1997). Según este autor, la estructura familiar se define como un conjunto invisible de demandas que organizan los modos en que interactúan los miembros de una familia. Es, además, un sistema que opera a través de pautas transaccionales, mantenidas por dos sistemas de coacción: el primero es genérico e implica las reglas universales de la organización familiar, por ejemplo, que un sistema funcional se organiza jerárquicamente basado en el poder, o que debe existir complementariedad de las funciones en los cónyuges. El segundo sistema de coacción es idiosincrásico e implica las expectativas mutuas de los diversos miembros.

Como sistema, la familia se compone de distintos subsistemas formados a partir de las relaciones que se dan dentro de ésta. Cabe mencionar, de esta forma, el subsistema parental, que Minuchin (1997) describe como la modificación que aparece con el nacimiento del primer hijo o hija. En esta situación, el subsistema conyugal debe diferenciarse para desempeñar tareas parentales, generando una transformación que debe trazar límites para el infante y los padres. A medida que este hijo crece, sus necesidades demandan modificaciones del subsistema parental, que debe adaptarse a los factores sociales que van apareciendo.

En cuanto al desarrollo cognitivo de este nuevo miembro, se destaca que los estilos, prácticas, competencias parentales y sus efectos influyen sobre éste. Vargas-Rubilar, y Arán-Filippetti (2014) constatan la importancia de la parentalidad en la maduración cerebral y desarrollo cognitivo, aunque éste es uno de tantos factores que influye en el proceso de crecimiento de un niño o niña y que, a su vez, estas competencias se encontrarán ligadas al entorno social de los progenitores.

En este contexto, el apoyo al desarrollo sociocognitivo y emocional de los niños requiere de una adecuada organización familiar y competencias parentales idóneas para nutrir emocional y socialmente el desarrollo de los niños generando un apego seguro. Al mismo tiempo, el ambiente social familiar funcional es un factor protector para el niño (Rutgers, et al, 2007).

\section{Interacción Familia y \\ Trastorno del Espectro Autista (TEA)}

El impacto del diagnóstico de TEA de un/a niño/a implica complejidades tanto a nivel familiar como para el sistema de salud pública. En la familia, es asumir una condición que afectará el curso de toda la vida del niño/a lo cual impacta en el proyecto- 
sistema familiar. Al mismo tiempo al ser un problema permanente se requiere de un sistema de salud que acompañe el desarrollo del niño en todas sus etapas (AcostaRodríguez, \& García, 2019). Los padres y el entorno familiar, poseen un valor primordial en el desarrollo de los individuos, siendo el primer grupo al que uno pertenece y el cual además incide en la adquisición de valores y bases para un progreso integral (Altarejos, Bernal, Buxarrais y Martínez, 2004), resultando comprensible que diversas actitudes comportamentales 0 variables psicológicas pudiesen influir en el clima, bienestar familiar y de la salud.

La presencia de enfermedad también puede influir en el clima y bienestar del grupo familiar. En este sentido, Armengol y Fuhrmann (1993) mencionan que:

"La enfermedad puede interrumpir el funcionamiento cotidiano de una familia y perturbarla en forma variable. Depende de la gravedad que atribuye a la enfermedad, del lugar que tiene el afectado en la familia, de la forma en que la sintomatología se inserta en el sistema de creencias y valores de la familia y del estado en el que se encuentra la organización" (p. 53).

Básicamente, todo depende de cómo se enfrenta la familia ante las distintas situaciones.

Así, podemos entender que las complicaciones derivadas de la enfermedad de un miembro no solo afectan el normal desarrollo de éste, sino que también impactan la organización de toda la familia. Podemos así tomar como ejemplo el caso de familias con un miembro con Síndrome de Down. Machín, Purón y Castillo (2009) mencionan que padres describen sensaciones de shock e incredulidad. Se intenta huir de la realidad y se dificulta la transmisión del diagnóstico a terceros, siendo normal negar la realidad porque la situación se percibe como insostenible.
En la actualidad, la Asociación Americana de Psiquiatría (2014) en el Manual de Diagnóstico Estadístico de Trastornos Mentales Quinta Edición (DSM-V) establece el concepto de TEA para hablar de un conjunto heterogéneo de alteraciones del neurodesarrollo cuya sintomatología definitoria incluye, además de un trastorno en el desarrollo sociocomunicativo y un patrón restringido de actividades e intereses, otro tipo de manifestaciones clínicas que varían enormemente de un individuo a otro (Martos-Pérez y Llorente-Comí, 2013). Estas alteraciones se encontraban distinguidas en Autismo Clásico, Síndrome de Asperger, Trastorno Desintegrativo Infantil, Síndrome de Rett, y un trastorno inespecífico generalizado del desarrollo (American Psychiatric Association, 1994). En Chile, y debido al uso de la Clasificación Internacional de Enfermedades, Décima Versión (Organización Mundial de la Salud, 1992), estas alteraciones, bajo el concepto de "Trastornos generalizados del desarrollo", continúan diferenciadas. Esto se traduce en el diagnóstico de síndromes y trastornos conceptualmente obsoletos y una desinformación generalizada sobre el TEA como tal, no así de las condiciones más diagnosticadas: el Autismo y el Síndrome de Asperger, de las cuales existen concepciones en la cultura general.

Espín, Cerezo y Espín (2013) señalan que el TEA es un "continuo de trastorno" ya que, si bien se presentan diversas manifestaciones clínicas, se caracteriza por el compromiso en tres ejes fundamentales: 1) Alteraciones cualitativas en la interacción social recíproca, 2) alteraciones en la comunicación verbal y no verbal, y 3) presencia de patrones restrictivos, repetitivos y estereotipados de la conducta (Wing, 1997).

En cuanto al diagnóstico, los síntomas asociados al TEA generalmente son reconocidos durante el segundo año de vida, pero pueden ser observados tempranamente si existe un retraso severo en el niño (Quijada, 2008). Estos síntomas se 
consolidarían a los 36 meses de edad (Melgar, Rangel \& Reynoso, 2017), aunque también puede detectarse incluso durante la adolescencia y la adultez.

Las alteraciones en cada una de las áreas afectadas en los diversos diagnósticos de TEA se presentan desde leves a severas. Sin embargo, las categorías diagnósticas inevitablemente implican definir un punto de corte, el cual puede llegar a limitar las expectativas de avance que poseen quienes rodean a la persona diagnosticada. Un TEA puede asociarse a diversos niveles intelectuales, habilidades de aprendizaje y características conductuales, que traen consigo desde dificultades sutiles hasta situaciones altamente discapacitantes (Ministerio de Salud, 2011).

La variabilidad en el diagnóstico y la sintomatología que puede presentarse hacen que resulte complejo poder generar consenso respecto al tratamiento. Sin embargo, un aspecto que es importante tener en cuenta, es la atención temprana en los niños con TEA; mientras antes inicie la intervención, mejores resultados pueden observarse (Clofent-Torrentó, HernándezLatorre, Montero-Camacho, Palau-Baduell, \& Salvadó-Salvadó, 2012).

La atención temprana es capaz de otorgar acciones que pueden facilitar un mayor nivel de desarrollo en lo personal y en la integración social, pudiendo reducir la gravedad de los síntomas del TEA en el niño (Sánchez-Raya, Martínez-Gual, Elvira, Luque, \& Alós, 2015). Si bien los programas de atención temprana requieren un modelo global de tratamiento, es prioritario enfocarse en la estimulación y desarrollo de habilidades socio-comunicativas y de juego por sobre los objetivos relacionados al desarrollo cognitivo o motor (Martos-Pérez \& Llorente-Comí, 2013; Melgar, Rangel \& Reynoso, 2017).

Al tener distintas necesidades, los niños con TEA requieren gran dedicación e implicación de los profesionales, y más aún de la familia, pues ésta constituye su entorno más cercano. Actualmente los programas de atención temprana la contemplan cada vez más en el proceso, dándoles recursos para optimizar el desarrollo de los niños, manejar situaciones que pueden resultar conflictivas, otorgándoles un papel activo en la toma de decisiones y guiando a los profesionales en las prioridades de las necesidades, pues ellos vivencian la realidad del trastorno día a día (Martos-Pérez \& Llorente- Comi, 2013).

Considerando la importancia del papel de la familia en un individuo, es relevante entender las implicaciones de ésta en el desarrollo educativo y social de los niños diagnosticados (Baña, 2015). En este sentido, las expectativas y comportamientos de los cuidadores tienen gran influencia en la intervención de quien padezca el trastorno. Por lo tanto, es importante considerar el impacto que tiene el diagnóstico en la unidad familiar ya que la presencia de un hijo con TEA genera en primera instancia un quiebre con las expectativas respecto a formar una familia. Es decir, se puede producir una pérdida de la significación del niño como persona normal para tener que asumir diversas limitaciones que le afectarán tanto en su desarrollo social como afectivo. Frente a esta situación se pueden dar diversas reacciones en la familia, tales como frustración, pena, negación o conflictos (Núñez, 2003; 2007).

A medida que el/a hijo/a va creciendo se pueden ir denotando más las alteraciones del desarrollo social y comunicativo, lo cual puede generar sentimientos de frustración, ansiedad y estrés en los cuidadores (Ramírez \& Mogollón, 2013). Según GarcíaFernández, Fernández, Sanjurjo, y Durán (2016), el $82 \%$ de los cuidadores de niños con TEA, presentan estrés parental con niveles clínicamente significativos, visualizando lo complejo de la situación y el impacto que genera en las familias.

La asimilación del diagnóstico en los padres se caracteriza por shock, 
inmovilización, depresión y aceptación de la realidad (Cuxart, 1998). Sin embargo, siguen ocurriendo cambios en el día a día familiar, como por ejemplo la restricción en la vida social, o adaptaciones en el entorno físico donde se encuentra el niño, pudiendo incrementar el aislamiento y la soledad en las familias (Martínez y Bilbao, 2008). De acuerdo Nuñez (2003), la pareja con hijo diagnosticado con discapacidad tiende a priorizar el vínculo parental por sobre el de pareja, a diferenciar rígidamente sus roles y con ello la aparición de conflictos que tienden a cronificarse.

Para comprender la experiencia de las familias que tienen un integrante diagnosticado con TEA se necesita un marco de referencia que permita acceder a los significados detrás de las vivencias. Esto puede ser accesible a través de las narrativas familiares en torno al diagnóstico.

\section{La Narrativa y su Estudio \\ de la Experiencia Familiar}

La narrativa es definida por Tarragona (2013) como el interés por la manera en la que las personas otorgan significado a sus vidas a través de narraciones o historias de su experiencia. Las historias generadas tienen gran importancia, porque transmiten el significado de sus vidas a la vez que organizan la forma en que experimentan las relaciones, la propia identidad y las posibilidades de la vida (Tarragona, 2008). Así, estas experiencias se reflejan en cómo sus narrativas cambian o se mantienen y así, en la medida que cambia la narrativa, también lo harían la percepción y la conducta.

La formación de historias construidas por una familia sobre alguna experiencia es una sinopsis de sus creencias, cultura, costumbres y narrativas compartidas. Una de las principales tareas desde la perspectiva narrativa es explorar éstas desde las diferentes realidades de los miembros de la familia y permitir que éstas sean compartidas y consensuadas entre sus miembros
(Rolland, 2000). Por su parte, Walsh (2004), señala que en los relatos también está la capacidad para configurar y reflejar los valores e intereses de nuestros tiempos, como también los significados que las personas les atribuyen: qué significa ser hombre o mujer, ser una familia, un padre, una madre entre otros.

Con las narrativas se construyen los significados para el individuo, siendo éstos el centro de la experiencia vital $y$, por ende, importantes para comprender el significado de la experiencia humana. Así las narrativas constituyen instrumentos elementales para la creación y comunicación del significado (Herrera \& Domínguez, 2013). Resulta importante comprender el significado de la experiencia, en tanto las personas vinculan éstas y sus acciones con significados específicos.

A su vez, las narrativas se adquieren en la relación social; esto debido a la naturaleza humana, la necesidad de interacción constante y al ser seres socialmente interdependientes. De este modo, las narrativas no adquieren significado en el interior de las personas, sino desde las relaciones establecidas con otros (Herrera \& Domínguez, 2013).

El carácter personal de las narrativas es particular, trayendo consigo emociones, sentimientos y motivaciones exclusivas de la persona (Herrera \& Domínguez, 2013). Smith y Sparkes (2008), señalan que no todas las narrativas pueden relatarse a todo el mundo, como tampoco pueden ser contadas de la misma forma. El investigador se convierte en coautor del relato, participando activamente en su creación (Riessman, 2008).

Según Herrera y Domínguez (2013), la narrativa es una forma de hablar de sí mismo, del pensamiento y de sus actos. Como enfoque de investigación ofrece la posibilidad de comprender tanto la vivencia de qué se vive en el tiempo y cómo se vive este tiempo. Temporalmente es una forma 
de recordar el pasado, configurar el presente y replantear el futuro.

A nivel nacional el TEA constituye parte de los planes de intervención tanto en los Ministerios de Salud como Educación (MINSAL, 2011; Mineduc, 2015). Sin embargo, por la complejidad del diagnóstico, en Chile sólo existen estadísticas parciales sobre su prevalencia y magnitud. Se estima que de 240.569 nacidos vivos registrados en el 2007 [DEIS36], la cantidad aproximada de personas con diagnóstico de TEA en Chile sería de 2156 niños. Asimismo, una de las discusiones actuales sobre la prevalencia del TEA es el sorpresivo aumento en los últimos años (MINSAL 2011).

Como señalamos anteriormente, el contexto social y familiar es fundamental en el apoyo al tratamiento. En Chile especialmente en la Región no existen suficientes estudios que aborden desde la perspectiva de la familia el impacto del diagnóstico y la vivencia de tener un miembro con diagnóstico de TEA, aspectos centrales al momento de promover un desarrollo sustentable para la persona que presenta dicho diagnóstico.

Este estudio busca en el contenido de las narrativas, el significado de las vivencias de los integrantes de la familia como un sistema complejo. En este caso busca conocer, no a un individuo y su visión, sino la perspectiva de los adultos significativos de la familia, siendo la pregunta: ¿Cuáles son las narrativas presentes en el discurso de los adultos significativos del grupo familiar en relación a tener un miembro diagnosticado con Trastorno del Espectro Autista?

Así, el objetivo general del presente estudio es: Describir las narrativas de los adultos significativos del grupo familiar con un miembro diagnosticado con Trastorno del Espectro Autista, a través de la vivencia de sus integrantes. Con esto, los objetivos específicos son los siguientes: a) Indagar acerca de los conocimientos e información que dichas personas tienen respecto al TEA, involucrando aspectos del tratamiento, desarrollo y conductas esperadas en la adolescente diagnosticada.

b) Explorar los sentimientos y emociones que comprenden y afectan la tarea de dichos miembros familiares a lo largo del proceso.

c) Reconocer características de las relaciones y del funcionamiento del grupo de adultos significativos.

\section{MÉTODO}

\section{Diseño}

Corresponde a un estudio cualitativo y exploratorio cuyo diseño es el estudio de caso único el cual se caracteriza por buscar la comprensión de la complejidad y la particularidad de casos singulares (Schwandt \& Gate 2018). De acuerdo a este diseño, el caso puede ser considerado un sistema complejo de procesos y debe ser abordado desde una perspectiva crítica, así el investigador está tratando con fenómenos reales o construidos, cuyos límites pueden ser físicos como también de interrelaciones (Byrne \& Callaghan, 2014, p 155). Este diseño fue seleccionado porque la familia es una unidad que posee una organización, donde se co-construyen narrativas sobre su realidad válida en sí misma y que representa formas específicas de abordar su problemática, como también ejemplifica el problema en estudio de una familia con características específicas.

\section{Participantes}

El caso corresponde a un grupo familiar urbano, de nivel socio-económico medioalto, de la ciudad de Temuco, región de La Araucanía, Chile. Es una familia nuclear, compuesta por el padre, de 50 años, con estudios universitarios y trabajador dependiente en el área de ventas. La madre, de 48 años, tiene estudios técnicos, se desempeña como dueña de casa, 
encargándose principalmente de las labores del hogar y del cuidado de la hija de 12 años diagnosticada con TEA. En la familia hay una tía materna de 24 años, con estudios universitarios, la cual también contribuye al cuidado de la niña. Los criterios de inclusión fueron: (a) que el grupo familiar tenga a su cuidado un miembro diagnosticado con una alteración englobada en el TEA, (b) que el miembro tenga el diagnóstico hace un año 0 más, y (c) que, desde el diagnóstico hasta la actualidad, quien presenta la alteración haya sido intervenido por profesionales respecto de su patología. El acercamiento a la familia fue por conveniencia a través de contacto personal con el equipo terapéutico.

\section{Procedimiento}

Recolectamos los datos mediante entrevistas cualitativas, individuales y grupal a la familia. Para las entrevistas se diseñó una pauta de preguntas abiertas basada en los objetivos específicos del estudio. La entrevista grupal a la familia se efectuó en base a preguntas circulares las que buscaban profundizar en la retroalimentación que otorga la familia a través de una sucesión de preguntas (Selvini-Palozolli, Boscolo, Cecchin \& Prata, 1980). La razón para aplicar esta entrevista a un grupo familiar, se fundamenta en la práctica de investigación familiar en salud, la cual toma a la familia como unidad de análisis buscando generar conocimientos de la relación del grupo con la salud de los integrantes y la población en conjunto (Louro \& Serrano, 2010).

Las entrevistas individuales y grupal de la familia fueron grabadas bajo consentimiento informado y transcritas para su posterior análisis. Se les informó y solicitó su participación en la investigación dando a conocer los resguardos éticos de anonimato, confidencialidad y voluntariedad. Se les propuso entrevistas familiares en el hogar, a fin de observarlos en un contexto con el que están familiarizados y sobre el cual tienen control, lo cual estimuló la confianza y comodidad de los participantes. En un tiempo aproximado de un mes, se realizaron tres visitas al hogar en horario vespertino, esto con el fin de poder tener la presencia de todos los miembros del sistema familiar, llevándose a cabo entrevistas de aproximadamente una hora y treinta minutos de duración.

\section{Estrategia de Análisis}

La estrategia de análisis se basó en una lógica inductiva, que permitió identificar, analizar y reportar temas recurrentes que emergieron de las propias narrativas de los entrevistados (Boyatzis, 1998). Braun y Clarke (2006), definen al tema como aquello que "captura algo importante sobre la información con relación al estudio, y representa algún nivel de significado dentro de los datos" (p.82). Los temas identificados reportan las vivencias, significados y la realidad de los miembros de la familia; y emergieron en función de su importancia, énfasis o recurrencia en el contexto de las narrativas.

La validación de los datos, fue a través de un proceso de triangulación que consideró las siguientes modalidades: 1) de investigadores, a fin de alcanzar un acuerdo intersubjetivo en relación a los datos registrados y al análisis de los significados que éstos adquieren (Denzin, \& Lincoln, 2017) 2) de técnica, utilizando entrevista grupal e individual lo cual contrasta la estabilidad de la información y 3) de fuente de información en que los distintos miembros de la familia aportaron al fenómeno en estudio, lo que permite la comprobabilidad de la información, verificando los datos mediante el consenso intersubjetivo.

\section{Hallazgos}

Presentamos los resultados en función de las tres categorías de análisis o temas que emergieron de las narrativas de los participantes, las cuales son: 1) conocimiento e información sobre el TEA, 2) afectividad y 3 ) dinámicas relacionales. Cada categoría es ejemplificada con citas de los 
participantes. Utilizamos seudónimos en aquellas citas en las que mencionan nombres.

\section{Categoría 1: Conocimiento e información sobre} TEA.

Corresponde al conocimiento e información que los cuidadores tienen respecto al TEA y hace referencia a la forma en que obtienen, definen y buscan la información relevante sobre el tema. Se evidenciaron como principales formas de adquisición los recursos entregados por los profesionales y especialistas, y las definiciones propias formadas desde la percepción y experiencia. La orientación profesional otorgó visiones y herramientas en el afrontamiento de la situación:

"...dijo que podíamos hacer un montón de cosas con ella, que igual iban a haber momentos en que no 10 íbamos a pasar bien, pero que ella iba a ir creciendo y que se iba a ir adaptando" Padre

Por su parte, las definiciones de TEA entregadas por los profesionales fueron sumamente relevantes en las concepciones que tienen actualmente del trastorno:

"Yo creo que lo más importante es 10 que me dijo el terapeuta ocupacional: 'Es un síndrome, no es una enfermedad... entrene a su hija y no va a tener ningún problema'..." Madre

También existió una búsqueda autónoma de información caracterizada por una iniciativa de informarse, la cual denota una preocupación respecto a la situación:

“... ¿Estará bien hecho el diagnóstico?, porque nos pusimos a leer, sobre todo Jessica (madre)... Entonces estaba en el umbral si era Asperger o puede ser autismo, entonces siempre pedimos una segunda opinión." Padre.
Además, los adultos significativos del grupo familiar contaron con definiciones propias acerca del TEA, algunas conceptualizadas desde su percepción y otras desde su experiencia.

"Lo otro, el vocabulario, a mí siempre me ha llamado la atención por ejemplo no es tierra, es lodo, es una cita, tu no vas salir o juntarte 0 algo, (...) utilizan palabras que a veces no saben el significado, eso sí me he dado cuenta..." Padre.

A su vez, los adultos contaron con información adquirida por medio de familiares y terceros, lo cual aportó a los conocimientos que poseían respecto a la situación.

“...Lucía (familiar), como ella es más profesional tiene como más... Y me decía, por ejemplo, que la Violeta (hija diagnosticada con TEA)... debieron orientarla en estrategias de estudio, en el orden de sus cuadernos, en darle tips para aprovechar su atención, (...) un terapeuta, no sé, alguien para superar la distracción (...) utiliza bien su tiempo, toda rutina, mezclar lo que le gusta con lo que no le gusta hacer, y dice: 'y así apuntar a su autonomía, por ejemplo, que aprenda a movilizarse sola" Madre.

\section{Categoría 2: Afectividad.}

La categoría Afectividad, se refiere a la respuesta emocional y sentimental de una persona hacia otra, o hacia un estímulo 0 situación. Ésta se ve reflejada en las respuestas que tienen ante los diferentes aspectos de sus vidas. Antes de tener un diagnóstico, la madre manifestaba sentimientos negativos de preocupación y frustración que le causaban tanto la situación como algunos comportamientos de su hija. Sin embargo, al recibir esta información aparecen reacciones emocionales más positivas. Lo primero que emerge es la 
sensación de alivio que los padres tuvieron ya que finalmente tenían una base sobre la cual trabajar en pos de comprender y ayudar a su hija. A partir de este momento en numerosas ocasiones los padres demuestran un compromiso constante con el proceso que conlleva la condición que ella tiene.

"Pero nosotros no nos quedamos conformes, entonces andamos, seguimos buscando, seguimos buscando, o sea si nos hubiésemos quedado con los primeros diagnósticos, ahí habríamos tenido problemas, pero seguimos, seguimos, seguimos" Padre.

Entre las consecuencias a partir del diagnóstico, la madre tuvo reacciones emocionales negativas, pues se encontró con que, a raíz de éste, la gente parecía tener un trato diferente con su hija. No consideraba que esto fuera correcto, puesto que su hija no siempre iba a tener una ventaja debido a su condición.

"Nos habian dicho que igual le podian hacer pruebas diferenciadas (...) pero, no, yo nunca estuve de acuerdo en el fondo, porque dije, la vida de aquí para adelante, para su futuro, o sea, no... El trabajo no va a ser así con ella, a ella le van a exigir y tiene que rendir" Madre.

La madre continuó con reacciones negativas ante los comportamientos de su hija, pues muchas veces le significaban una sobrecarga emocional y de responsabilidades. También expresaba molestia y vergüenza por algunas conductas no aceptadas socialmente.

"Me hacía pasar unas vergüenzas con las cosas que decía (...) me hacía preguntas donde no era el lugar ni el momento (...) subíamos al colectivo y yo me empezaba a poner nerviosa (...) entonces va y dice: "mamá, ¿Por qué mis compañeros tienen el pene tan chiquitito y no 10 tienen grande como mi papá?" (...) me quería bajar y todos muertos de la risa... yo dije ¡Qué vergüenza!" Madre.

Con respecto a la actitud de los padres hacia el futuro de su hija, se pudo observar que la posible dificultad de ella para adaptarse les preocupa a ambos, puesto que saben que no podrán estar siempre para ayudarla. Sin embargo, tienen expectativas positivas respecto a su futuro, pues saben que tiene la capacidad intelectual para llegar lejos.

"Entonces a futuro (...) yo creo que le va a ir bien. Ahora, no sé... no me la imagino a los diecisiete años en la universidad, con lo inmadura... porque ella es muy inmadura..." Madre.

\section{Categoría 3: Dinámicas relacionales.}

Definimos como dinámicas relacionales al vínculo entre dos o más personas, el cual está susceptible a cambios y a reorganizaciones en sus miembros y roles, viéndose afectado por cada una de sus partes. Se percibe en la familia una evidencia de lazos fuertes, expresado por la unión que sienten sus padres entre ellos y en torno a su hija.

"Si, nosotros todo lo que hacemos, 10 hacemos por ella, todo es ella, nuestro mundo es ella" Padre.

"Yo creo que como que hubo más unión, más unión que en vez de alejarnos o de culparnos de que lo hicimos mal, que por tu culpa tú eres así, a lo mejor lo heredó de ti, no sé, no, no, nada" Madre.

Este vínculo no existe solo entre los padres y su hija, sino que la tía también tiene un rol importante dentro del desarrollo de su 
sobrina, siendo la más cercana, percibiendo ella también el apego entre ambas.

"...La Violeta, además de ser como sobrina y tía, somos bien amigas, entonces siempre ella me cuenta todas sus cosas de, no sé, o lo que le inquieta o me pregunta, me cuenta, cosas que le importan a ella, y... no sé, yo creo que me debe considerar cercana, por la confianza, el cariño, sus muestras de afectos, ese tipo de cosas..."Tía.

También se pueden distinguir dificultades a causa de la presencia del diagnóstico de TEA, como el conflicto para que realice actividades fuera de sus intereses comunes, dificultades a la hora de comer por mostrarse demasiado selectiva y una serie de conflictos entre madre-hija, debido a que es la madre quien está a cargo de la adolescente y del hogar. El padre, por su parte ejerce un rol principalmente de proveedor, lo cual no atenta a la unidad familiar ya que se señala que ambos cumplen roles complementarios.

"Yo hago todo, yo me preocupo de todo el tema de la Violeta, de la casa, él trabaja [...], igual como que me gustaría que él me acompañara, pero yo sé cómo es su trabajo también, y que gracias a eso podemos brindarle todos estos tipos de apoyo a la Violeta" Madre

Ambos padres aplican un rol parental directivo, en el que ordenan a su hija realizar determinada conducta, logrando un mejor resultado que al preguntarle su opinión. También intentan entregarle enseñanzas para su adaptación pensando en las posibles dificultades que puede presentar a causa del TEA.

"Teníamos que entrenarla para el mundo real, para lo que a ella le va a tocar vivir, enfrentar y sacar todas esas carencias que ella tiene..." Padre.
Por su parte, la adaptación al ámbito escolar se ha visto mermada debido a que el colegio no tendría las herramientas necesarias para educar a estudiantes con TEA, no contando con profesionales especializados y con información pertinente, destacándose la poca adaptación en el aula y la baja aceptación del trastorno por parte de algunos profesores.

"La profesora de matemáticas (...) una vez que la Violeta no entendía un ejercicio, y le pidió a la profesora que se lo explicara y ella le dijo que a ella no le pagaban por explicarle a niñitas especiales (...) La Violeta no me dijo nada, y creo que se puso a llorar, calladita, y siguió, y me contaron los papás porque los niños llegaron diciendo a la casa "que en la clase..." y que la profesora había dicho a la Violeta y todo y nosotros fuimos a reclamar" Madre.

\section{DISCUSION}

El estudio logra describir tres categorías que revelan las narrativas de esta familia respecto a las vivencias de tener un miembro con diagnóstico de TEA. Estos hallazgos ejemplifican la experiencia de una familia chilena con nivel socioeconómico medio alto en la que se observan: sus conocimientos e información acerca del problema, sus sentimientos y emociones y las características de sus relaciones y funcionamiento como grupo familiar. Se observan marcados roles de género siendo la madre quien se ocupa de los cuidados directos de la niña y el padre, una figura proveedora de recursos económicos, pero que logran complementarse de modo sinérgico.

Llama la atención que el conocimiento e información de los adultos respecto al TEA provenga de los profesionales, manifestándose como un pilar de gran importancia en su experiencia, pudiendo adquirir conocimientos y conductas que facilitaron su proceso, así como 
herramientas para situaciones futuras. Se destaca orientación y apoyo profesional, manejo conceptual, definiciones profesionales y de reconocimiento sobre el TEA.

En esta misma categoría, se observaron definiciones propias sobre el TEA, las cuales emergieron desde la vivencia misma de la familia. Las narraciones de estas experiencias familiares son el vehículo por el cual los miembros le otorgan significado a sus vidas, lo cual a su vez guarda relación con que las narrativas adquieren significado desde las relaciones establecidas con otros y no desde la individualidad de las personas (Herrera \& Domínguez, 2013; Tarragona, 2013).

Otra forma de adquisición de información fue a través de procesos de búsqueda autónoma, surgidos por iniciativa propia y en menor medida información adquirida durante sus procesos educativos. A su vez, esta adquisición se reforzó a través de la comunicación de su propio problema hacia la red de amigos y familiares lo que coincide con Herrera y Domínguez (2013) al afirmar estos que las narrativas son formadas como instrumentos elementales para la comunicación del significado a otros. En síntesis, se observó que el manejo de información y la conceptualización del trastorno se generan en un proceso mixto entre la relación con los profesionales y la vivencia misma de la familia, lo que es un hallazgo de esta investigación.

En el grupo familiar estudiado, se denotan emociones negativas ligadas principalmente a la preocupación constante de la madre por su hija y a su frustración, sin embargo, surgen reacciones emocionales positivas asociadas al alivio y tranquilidad que sintieron los padres al recibir el diagnóstico, lo cual no concuerda con la literatura existente que señala un quiebre con las expectativas existentes al momento de recibir el diagnóstico (Ramírez, O. y Mogollón D; Machín, Purón \& Castillo, 2009).
Se observa además como una narrativa constante el gran compromiso que tienen con la situación de su hija, lo cual coincide con antecedentes empíricos sobre los cambios en el contexto familiar que se dan al momento de recibir el diagnóstico (Martínez \& Bilbao, 2008).

Tanto la proactividad como los cuidados que tienen con su hija, se constituyen como factores protectores de la niña. En ese sentido, se aprecia la gran dedicación como un aspecto que facilita el proceso puesto que favorece la búsqueda de información, la atención con profesionales y una mejor predisposición en dinámicas relacionales; aunque también existen consecuencias complejas para la familia tales como el aislamiento en relación a sus amistades.

Por otra parte, se evidencia que es la madre quien presenta la mayor sobrecarga emocional, ya que pasa más tiempo con la niña y es su cuidadora principal, siendo además la encargada de administrar el área económica del hogar. En ella se vio como una narrativa dominante las reacciones emocionales negativas ante el comportamiento de la niña, dificultando la comprensión de sus conductas. Con una intervención más temprana pudieran haberse observado mejores resultados, tanto en la joven diagnosticada, como en la familia, favoreciendo así el entendimiento del comportamiento y un mejor manejo de éste (Sánchez-Raya et al., 2015) Al considerar que cada vez los programas de atención de TEA incluyen más a la familia, otorgándoles un rol más activo (Llorente-Comi, \& Martos Pérez, 2013), es necesario que se incluya a toda la familia en procesos de apoyo y orientación, ya que se ve a la madre más estresada como suele ocurrir con cuidadores de personas con TEA (García-Fernández, Fernández, Sanjurjo, \& Durán, 2016).

La preocupación por el futuro de la adolescente les genera ansiedad, no saber cómo podría ser la vida de su hija sin ellos, 0 cómo va a desenvolverse en contextos 
distintos a los habituales. Según Rodríguez (2006), a medida que los hijos van creciendo se pueden apreciar cambios tanto comunicacionales como sociales, pudiendo causar sentimientos de estrés 0 ansiedad en los cuidadores. Esta narrativa puede asociarse al gran compromiso parental, ya que, al sentirse tan implicados en el proceso, se les dificulta imaginar un futuro donde ellos no pudiesen ayudarla y acompañarla.

Se puede agregar que las diferencias de esta familia respecto a lo señalado por la bibliografía (Cuxart, 1998; Martínez \& Bilbao, 2008), se entienden desde la mirada construccionista de Armengol y Fuhrmann (1993), señalando lo relativo que puede ser el impacto de una enfermedad en la familia, debido al contexto que le rodea, las creencias implícitas y la manera en que ésta se encuentra organizada. Así, lo evidenciado en los adultos significativos del grupo familiar estudiado es uno de los posibles escenarios que pueden darse. La notificación del diagnóstico generó en la familia un alivio, ya que se pudo comprender qué aspectos de la conducta de la hija, que preocupaba a los padres, eran propios del Trastorno del Espectro Autista. La presencia de fuertes lazos dentro de la familia nuclear, la unión entre los padres y el apoyo de una tía cercana son factores que han facilitado el proceso de adaptación y que la misma familia reconoce como positivo. Según Baña (2015), el hijo con TEA requiere de muchas más necesidades y demandas de apoyo personal y social y necesita superar más situaciones complejas a lo largo de su desarrollo, siendo la familia un factor que puede tanto aliviar como agravar la tensión en ésta. Las dinámicas de este grupo familiar atenúan las dificultades que la adolescente ha presentado en su desarrollo, siendo influido por la buena recepción de la información obtenida y el contexto en el que ésta se dio.

No obstante, se observan algunas dificultades relacionales en la familia, específicamente en la díada madre-hija. Ramírez y Mogollón (2013) señalan que los cuidadores pueden sentir frustración, ansiedad y estrés a raíz de las alteraciones del desarrollo que presenta el TEA, lo que puede observarse en la madre de la menor, la cual, al tener un marcado rol de cuidadora dentro de la familia, vive en mayor medida el impacto del diagnóstico y experimenta mayor estrés. Se corrobora también la relación entre calidad de vida, estrés parental y rol de cuidador hacia un hijo diagnosticado con TEA (García-Fernández, Fernández, Sanjurjo, \& Durán, 2016).

Por otra parte, se aprecian dos principales recursos asociados a la crianza, siendo el primero de estos el rol parental directivo caracterizado por una adecuación del lenguaje hacia la adolescente con el fin de asegurar el entendimiento por parte de ella y evitar malinterpretaciones. En segundo lugar, una enseñanza centrada en la adaptación a otros contextos y situaciones. En este sentido, Pillcorema (2013) nos recalca el papel central que juega la familia en la regulación y canalización de significados sociales y culturales en la vida de las personas, viéndose esto reflejado en el intento de la familia de que su hija se adapte a su entorno, pudiendo así desempeñarse de mejor manera en los diferentes espacios presentes y futuros.

En esta línea, se observa que la familia ha empleado mecanismos que han guiado su objetivo de educar de forma integral a su hija, lo cual ha sido gracias también a los programas de atención que recibieron, los cuales les entregaron herramientas y una mayor comprensión de lo que significa el trastorno. En este sentido, es necesario destacar el aporte que pueden generar los programas de atención temprana, los cuales tienen un gran impacto en el desarrollo de los menores, intentando entregar los recursos y herramientas necesarias para potenciar y optimizar el proceso evolutivo de los niños (Clofent-Torrentó, et al., 2012; LlorenteComi, \& Martos-Pérez, 2013; Sánchez-Raya, et al., 2015). 
Es importante destacar el rol que desempeñan los profesionales en el trabajo no sólo con el paciente diagnosticado, sino con todo el sistema familiar, siendo de gran importancia la confianza y la relación que se genera en este vínculo familia-profesional, aspecto que sin lugar a duda tiene el potencial de mejorar las condiciones de vida, brindar herramientas y apoyo hacia el futuro.

En relación con las limitaciones del presente estudio, se encuentra el hecho de que se abordó la vivencia sólo del núcleo familiar, sin expandirse a más miembros de ésta, lo que no permite corroborar algunos datos importantes expresados por los entrevistados e impide la probabilidad de encontrar nuevas referencias al no recabar información desde la familia extensa de la adolescente.

Como futuras líneas de investigación sería interesante comparar varias vivencias familiares, utilizando como variables el nivel socioeconómico o educacional, los cuales pueden ser factores de riesgo que afecten la capacidad de la familia para brindar apoyo al niño. Por otra parte, se recomienda ampliar la cantidad de miembros entrevistados para tener una visión más holística del caso a tratar. En esta misma línea, sería ideal incluir la perspectiva que pueden entregar los profesionales tratantes, puesto que, desde los hallazgos encontrados en el presente estudio, éstos se constituyen como un elemento prioritario en el tratamiento del TEA, por lo que su visión y experiencia al respecto sería sumamente relevante. Además de éstos, sería oportuno contar con datos entregados por parte del colegio en que se encuentre inserto, en caso que el paciente índice se encuentre en etapa escolar.

Como recomendación para futuros investigadores se propone la elaboración de investigaciones mixtas que permitan obtener información cuantitativa generalizable para un número más amplio de personas, pero sin perder de vista la necesidad de profundizar en la vivencia de los miembros familiares.
Finalmente, a partir de los hallazgos de este estudio, es recomendable para la familia con un miembro con TEA mantener una asesoría permanente con un equipo especializado evitando el aislamiento y fortaleciendo la capacidad de acogida, comprensión y desarrollo de recursos de apoyo.

\section{REFERENCIAS}

Acosta-Rodríguez, A. \& García Rohena, M. (2019). Autismo, transición y familia. En D. Pérez Jiménez, A. Rodríguez, I. Serrano-García, J. Serrano, R. Díaz Juarbe, \& S. Pérez López, (Eds.) Desarrollo humano: Travesía de Oportunidades y Retos. (pp. 227-248) Humacao, Puerto Rico: Artes Gráficas, Universidad de Puerto Rico.

Altarejos, F., Martínez, M., Buxarrais, M.R. \& Bernal, A. (2004). Familia, valores y educación. En M.A. Santos y J.M. Touriñán (Eds.). Familia, Educación y Sociedad Civil (pp. 89-136). Santiago de Compostela: Universidad de Santiago de Compostela.

Armengol, V. \& Fuhrmann, I. (1993). Enfermedad - organización familiar: una interacción compleja. De Familias y Terapias, 1(2), 53-61.

American Psychiatric Association (1994). Diagnostic and Statistical Manual of Mental Disorders (DSM-IV) (4th Ed.). Washington, DC.

Asociación Americana de Psiquiatría. (2014). Manual Diagnóstico y Estadístico de los Trastornos Mentales (DSM-V) (5ta Ed). España: Editorial Médica Panamericana.

Baña, M. (2015). El rol de la familia en la calidad de vida y la autodeterminación de las personas con Trastorno del Espectro del Autismo. Ciencias Psicológicas, 9(2), 323-336.

Bernales, S. (1993). La familia chilena actual ¿Cuál familia? Revista De Familias y Terapias, 1(2), 62-78.

Boyatzis, R. (1998). Transforming Qualitative Information: Thematic Analysis and 
Code Development. Thousand Oaks, CA: Sage Publications.

Braun, V. \& Clarke, V. (2006). Using thematic analysis in psychology. Qualitative Research in Psychology, 3, 77-101.

Bruner, E. (1986). Ethnography as Narrative. Chicago: University of Illinois Press.

Byrne, D., \& Callaghan, G. (2014). Complexity Theory and the Social Sciences. London: Routledge.

Clofent-Torrentó M., Hernández-Latorre M., Montero-Camacho M., Palau-Baduell M., Salvadó-Salvadó B. (2012). Modelos de intervención global en personas con trastorno del espectro autista. Revista de Neurología; 54(Supl 1): S63-71.

Cuxart, F. (1998). La familia del niño con autismo y el apoyo terapéutico a las familias. En A. Riviere \& J. Martos (Eds.). Tratamiento de Autismo. Nuevas perspectivas. (pp. 173-188). Madrid: Editores Instituto de Migraciones y Servicios Sociales.

Denzin, N., \& Lincoln, Y. (2017). The SAGE Handbook of Qualitative Research. (5th Ed.). California: Sage.

Espín, J., Cerezo, M., \& Espín, F. (2013). Lo que es trastorno del espectro autista y lo que no lo es. Anales de Pediatría Continuada, 11(6), 333-341.

García-Fernández, M., Fernández, J. C., Sanjurjo, B., \& Durán, M. (2016). Afrontamiento, estrés parental y calidad de vida de los cuidadores principales de personas con TEA. Revista de Estudios e Investigación en Psicología y Educación, 3(1), 60-68.

Giné, C. (2001). Familia e intervención precoz. Orientaciones para la intervención del psicopedagogo. En B. Del Rincón (Ed.), Presente y Futuro del Trabajo Psicopedagógico. Barcelona: Ariel.

Stake, R. (1999). Investigación con Estudio de Casos (2nd Ed). Madrid: Ediciones Morata.

Herrera, J. D. \& Domínguez, E. (2013). La investigación narrativa en psicología: definición y funciones. Psicología desde el Caribe, 30(3), 620-641. http://www.scielo.org.co/scielo.php?scri pt=sci_arttextypid=S0123417X2013000300009

Louro, I. \& Serrano, A. (2010). La investigación familiar y el valor de la metodología cualitativa para el estudio del afrontamiento a la enfermedad sicklemica. Revista Cubana de Salud Pública, 36(1), 37-53.

Machín, M., Purón, E. \& Castillo, J. (2009). Reflexiones sobre la intervención temprana en niños con Síndrome de Down considerando la familia y la comunidad. Revista Habanera de Ciencias Médicas, 8(1), 1-7.

Martos-Pérez J. \& Llorente-Comí M. (2013). Tratamiento de los trastornos del espectro autista: unión entre la comprensión y la práctica basada en la evidencia. Revista de Neurología, 57 (1), 185-191.

Martínez, M. \& Bilbao, M. (2008). Acercamiento a la realidad de las familias de personas con autismo. Psychosocial Intervention, 17(2), 215230.

Melgar, V., Rangel, M., \& Reynoso, C. (2017). El Trastorno del Espectro Autista: aspectos etiológicos, diagnósticos y terapéuticos. Revista Médica Del Instituto Mexicano Del Seguro Social, 55(2), 214-223.

Ministerio de Educación de Chile (2015). Ley 20.845 de Inclusión Escolar. Recuperado de http://www.rmm.cl/sites/default/files/ley20845_08-jun-2015.pdf

Ministerio de Salud (2011). Guía de Práctica Clínica de Detección y Diagnóstico oportuno de los Trastornos del Espectro Autista (TEA). Recuperado de: http://web.minsal.cl/portal/url/item/bd81 e3a09ab6c3cee040010164012ac2.pdf

Minuchin, S. (1997). Familias y Terapia Familiar. Barcelona: Gedisa.

Núñez B.A. (2007). Familia y Discapacidad: De la Vida Cotidiana a la Teoría. Editorial Lugar. 
Núñez, B. (2003). La familia con un hijo con discapacidad: sus conflictos vinculares. Archivos Argentinos de Pedriatría, 101(2), 133-142.

Organización Mundial de la Salud (1992). Clasificación Internacional de Enfermedades, Décima Versión (CIE10).

Pillcorema, B. (2013). Tipos de familia estructural y la relación con sus límites (Tesis, Universidad de Cuenca, Cuenca, Ecuador). Recuperada de http://dspace.ucuenca.edu.ec/bitstream /123456789/4302/1/Tesis.pdf

Quijada G, C. (2008). Espectro autista. Revista Chilena de Pediatría, 79(1), 8691.

Ramírez, O. \& Mogollón D. (2013). El estrés de crianza en madres de hijos con la condición de autismo severo. Revista Multidisciplinaria Dialógica, 10(1),66-93 http://revistas.upel.edu.ve/index.php/dia logica

Riessman, C. (2008). Narrative Methods for the Human Sciences. California: Sage.

Rolland, J. (2000). Familias, Enfermedad y Discapacidad. Barcelona: Gedisa.

Rutgers, A, ljzendoorn, M., BakermansKranenburg, M., Swinkels, S., Daalen, E., Dietz,C., Naber, F., Buitelaar, J. \& Engeland, H. (2007). Autism, attachment and parenting: $A$ comparison of children with autism spectrum disorder, mental retardation, language disorder, and non-clinical children. Journal of Abnormal Child Psychology, 35, 859-870.

Sánchez-Raya, M. A., Martínez-Gual, E., Elvira, J. A., Luque, B., \& Alós, F. (2015). La atención temprana en los Trastornos del Espectro Autista (TEA). Psicología Educativa, 21(1), 55-63. https://doi.org/10.1016/j.pse.2014.04.00 1.

Schwandt, T. \& Gates, F. (2017) Case study methodology. En N. Denzin, \& $Y$. Lincoln (Eds.) The SAGE Handbook of Qualitative Research (pp. 600-630). California: Sage.
Selvini-Palozolli, Boscolo, L., Cecchin, G. \& Prata, G. (1980). Hipotetización circularidad - neutralidad. Tres guías para el conductor de la sesión. Family Process, 19, 3-12.

Smith, B. \& Sparkes, A.C. (2008). Contrasting perspectives on narrating selves and identities: An invitation to dialogue. Qualitative Research, 8(1) 515.

Tarragona, M. (2008). Postmodern/ postestructuralist therapy. En J. Lebow (Ed.), 21st Century Psychotherapies (2da Ed.). Hoboken: Wiley y Sons.

Tarragona, M. (2013). Psicología positiva y terapias constructivas: una propuesta integradora. Terapia Psicológica, 31(1), 115-125.

https://doi.org/10.4067/S071848082013000100011

Vargas-Rubilar, J. \& Arán-Filippetti, V. (2014). Importancia de la Parentalidad para el Desarrollo Cognitivo Infantil: una Revisión Teórica. Revista Latinoamericana de Ciencias Sociales, Niñez y Juventud, 12(1), 171-186.

Walsh, F. (2004). Resilencia Familiar: Estrategias para su Fortalecimiento. Buenos Aires: Amorrortu.

Willig, C. (2013). Introducing Qualitative Research in Psychology (3ra Ed.). Maidenhead: McGraw Hill Education.

Wing, L. (1997). The autistic spectrum. Lancet, 350(9093), 1761-1766.

Todos los trabajos publicados en Revista Salud \& Sociedad (ISSNe:0718-7475) están sujetos a una licencia Creative Commons Reconocimiento 4.0 Internacional

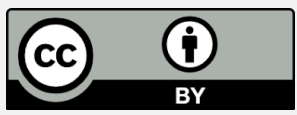




\section{RESUMO}

Objetivo: 0 propósito desta pesquisa foi descrever a experiência de adultos significativos de um grupo familiar que convivem com um membro diagnosticado com uma alteração do desenvolvimento que faz parte do Transtorno do Espectro Autista (TEA). Método: Como técnica de coleta de dados foi utilizada a entrevista grupal focalizada na família que compõe a amostra, sob um design de caso exclusive. Os resultados foram obtidos por meio da análise temática, nos eixos de Conhecimento e Informação, Afetividade e Dinâmicas Relacionais. Resultados: Entre as descobertas relevantes está o fato de que as mudanças produzidas pelo diagnóstico no grupo familiar não condizem com a teoria, uma vez que não se produziu uma crise ao receber o diagnóstico, mas um alívio relacionado a possibilidade de compreender a realidade. De todo modo, a família reconhece como relevante 0 apoio profissional e a informação oferecida pelos especialistas. Conclusão: é aconselhável que a família com um membro com TEA mantenha aconselhamento permanente com uma equipe especializada, evitando 0 isolamento e fortalecendo a capacidade de receber, entender e desenvolver recursos de apoio.

PALAVRAS-CHAVE: Narrativa familiar, Trastorno do Espectro Autista, TEA, família.

\section{RESUMEN}

Objetivo: El propósito de esta investigación fue describir la experiencia de los adultos significativos de un grupo familiar que conviven con un miembro diagnosticado con una alteración del desarrollo que forma parte del Trastorno del Espectro Autista (TEA). Método: Como técnica de recolección de datos utilizamos la entrevista grupal focalizada a la familia que compone la muestra, bajo un diseño de caso único. Obtuvimos los resultados a través de un análisis temático, utilizando tres códigos principales: conocimiento e información, afectividad, y dinámicas relacionales. Resultados: Como hallazgos relevantes identificamos que los cambios producidos por el diagnóstico en el grupo familiar no se condicen con la teoría, dado que no se produjo una crisis al enterarse de dicho diagnóstico, sino más bien un alivio ligado a la posibilidad de comprender la realidad. Asimismo, la familia reconoce como relevante el apoyo profesional y la información entregada por los especialistas. Conclusión: es recomendable para la familia con un miembro con TEA mantener una asesoría permanente con un equipo especializado, evitando el aislamiento y fortaleciendo la capacidad de acogida, comprensión y desarrollo de recursos de apoyo.

PALABRAS CLAVE: Narrativa familiar, Trastorno del Espectro Autista, análisis temático, familia. 